\title{
UEBER FLÄCHEN VON CONSTANTER GAUSSSCHER KRÜMMUNG*
}

\author{
VON \\ DAVID HILBERT
}

\section{Ueber Flächen von negativer constanter Krümmung.}

Nach BeLtramı $\dagger$ verwirklicht eine Fläche von negativer constanter Krümmung ein Stück einer LobatschefskiJschen (nicht-EukLIDischen) Ebene, wenn man als Gerade der LobatschefsKiJschen Ebene die geodätischen Linien der Fläche von constanter Krümmung betrachtet und als Längen und Winkel in der LoвatscheFsKIJschen Ebene die wirklichen Längen und Winkel auf der Fläche nimmt. Unter den bisher untersuchten Flächen negativer constanter Krümmung finden wir keine, die sich stetig und mit stetiger Aenderung ihrer Tangentialebene in der Umgebung jeder Stelle überall hin ausdehnt; vielmehr besitzen die bekannten Flächen negativer constanter Krümmung singuläre Linien, über die hinaus eine stetige Fortsetzung mit stetiger Aenderung der Tangentialebene nicht möglich ist. Aus diesem Grunde gelingt es mittelst keiner der bisher bekannten Flächen negativer constanter Krümmung, die ganze LobatschefskiJsche Ebene zu verwirkliçhen, und es erscheint uns die Frage von principiellem Interesse, ob die GANZE LOBaTschefskiJsche Ebene überhaupt nicht durch eine analytische Fläche negativer constanter Kr ̈̈̈mmung auf die Beltramische Weise zur Darstellung gebracht werden kann.

Um diese Frage zu beantworten, gehen wir von der Annahme einer analytisehen Fläche der negativen constanten Krümmung -1 aus, die im Endlichen überall sich regulär verhält und keine singulären Stellen aufweist; wir werden dann zeigen, dass diese Annahme auf einen Widerspruch führt. Eine solche Fläche, wie wir sie annehmen wollen, ist durch folgende Aussagen vollständig charakterisirt :

Jede im Endlichen gelegene Verdichtungstelle von Punkten der Fläche ist ebenfalls ein Punkt der Fläche.

Bedeutet $O$ irgend einen Punkt der Fläche, so ist es stets möglich, das rechtwinklige Coordinatenkreuz $x, y, z$ so zu legen, dass $O$ der Anfangspunkt des Coordinatensystems wird und die Gleichung der Fläche in der Umgebung dieses Punktes $O$ wie folgt lautet :

\footnotetext{
* Presented to the Society, October 27, 1900. Received for publication October 9, 1900.

† Giornaledi Matematiche, Bd. 6, 1868.
} 


$$
z=a x^{2}+b y^{2}+\mathfrak{P}(x, y),
$$

wo die Constanten $a, b$ die Relation

$$
4 a b=-1
$$

befriedigen und die Potenzreihe $\mathfrak{P}(x, y)$ nur Glieder dritter oder höherer Dimension in $x, y$ enthält. Offenbar ist dann die $z$-Axe die Normale der Fläche und die $x$ - und $y$-Axe geben die Richtungen an, die durch die Hauptkrümmungen der Fläche bestimmt sind.

Die Gleichung

$$
a x^{2}+b y^{2}=0
$$

bestimmt die beiden Haupttangenten der Fläche durch den Punkt $O$ in der $x y$-Ebene; dieselben sind daher stets von einander getrennt und geben die Richtungen an, in denen die beiden Asymptotencurven der Fläche durch den beliebigen Punkt $O$ verlaufen. Jede dieser Asymptotencurven gehört einer einfachen Schaar von Asymptotencurven an, die die ganze Umgebung des Punktes $O$ auf der Fläche regulär und lückenlos überdecken. Verstehen wir daher unter $u, v$ genügend kleine $\mathrm{Werthe}$, so können wir gewiss folgende Construktion ausführen. Wir tragen auf einer der beiden durch $O$ gehenden Asymptotencurven den Parameterwerth $u$ von $O$ als Länge ab, ziehen durch den erhaltenen Endpunkt die andere mögliche Asymptotencurve und tragen auf dieser den Parameterwerth $v$ ab: der nun erhaltene Endpunkt ist ein Punkt der Fläche, der durch die Parameterwerthe $u, v$ eindeutig bestimmt ist. Fassen wir demgemäss die rechtwinkligen Coordinaten $x, y, z$ der Fläche als Functionen von $u, v$ auf, indem wir setzen .

$$
x=x(u, v), \quad y=y(u, v), \quad z=z(u, v),
$$

so sind diese jedenfalls für genügend kleine $\mathrm{W}$ erthe von $u, v$ reguläre analytische Functionen von $u, v$.

Die bekannte Theorie der Flächen von der constanten Krümmung - 1 liefert uns ferner die folgenden Thatsachen :

Bedeutet $\varphi$ den Winkel zwischen den beiden Asymptotencurven durch den Punkt $u, v$, so erhalten die drei Fundamentalgrössen der Fläche die Werthe :

$$
\begin{aligned}
& e \equiv\left(\frac{\partial x}{\partial u}\right)^{2}+\left(\frac{\partial y}{\partial u}\right)^{2}+\left(\frac{\partial z}{\partial u}\right)^{2}=1, \\
& f \equiv \frac{\partial x}{\partial u} \frac{\partial x}{\partial v}+\frac{\partial y}{\partial x} \frac{\partial y}{\partial v}+\frac{\partial z}{\partial u} \frac{\partial z}{\partial v}=\cos \varphi \\
& g \equiv\left(\frac{\partial x}{\partial v}\right)^{2}+\left(\frac{\partial y}{\partial v}\right)^{2}+\left(\frac{\partial z}{\partial v}\right)^{2}=1 ;
\end{aligned}
$$


und mithin wird das Quadrat der Ableitung der Bogenlänge einer beliebigen

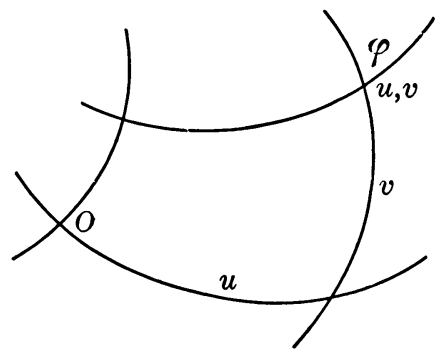

Fia. 1.

Curve auf der Fläche nach einem Parameter $t$ von der Form:

$$
\left(\frac{d s}{d t}\right)^{2}=\left(\frac{d u}{d t}\right)^{2}+2 \cos \varphi \frac{d u}{d t} \frac{d v}{d t}+\left(\frac{d v}{d t}\right)^{2}
$$

Der Winkel $\varphi$ genügt als Function von $u, v$ der partiellen Differentialgleichung

$$
\frac{\partial^{2} \varphi}{\partial u \partial v}=\sin \varphi \text {. }
$$

Die Formel (2) beweist den bekannten Satz :*

In jedem Vierecke, das von vier Asymptotencurven unserer Fläche gebildet wird, sind die gegenüberliegenden Bogen einander gleich.

Die Formel (3) gestattet die Berechnung des Flächeninhaltes eines von Asymptotencurven gebildeten Viereckes mittelst seiner Winkel; Darboux $\dagger$ ist auf diesem Wege zu dem folgenden Satze gelangt :

Der Flächeninhalt eines aus Asymptotencurven gebildeten Viereckes auf unserer Fläche ist gleich der Summe der Winkel des Viereckes vermindert um $2 \pi$.

Die Formeln (1) liefern eine Parameterdarstellung unserer Fläche, bei welcher die Coordinatenlinien

$$
u=\text { const. }, \quad v=\text { const } .
$$

die Asymptotencurven sind. Nach den obigen Ausführungen erweisen sich die rechtwinkligen Coordinaten $x, y, z$ gewiss für genügend kleine Werthe von $u, v$ als umkehrbar eindeutige Functionen der Variabeln $u, v$ d. h. die Formeln (1) vermitteln jedenfalls die umkehrbar eindeutige Abbildung eines Stückes der $u v$-Ebene in der Umgebung des Punktes $u=0, v=0$ auf ein Stïck unserer Fläche in der Umgebung des Punktes $O$. Unsere Aufgabe besteht darin, die

* DinI, Annalidi Mathematica, Bd. 4, 1870, S. 175.

Darboux, Leçons sur la théorie générale dessurfaces, Bd. 3, No. 773.

BranchI, Lezioni digeonetria differenziale, $\$ 67$.

†loc. cit., Bd. 3, No. 773. 
gesammte Abbildung der $u v$-Ebene auf unsere Fläche zu untersuchen, welche durch die analytische Fortsetzung der Formeln (1) erhalten wird.

Fassen wir irgend eine Asymptotencurve unserer Fläche ins Auge, so erkennen wir sofort, dass dieselbe im Endlichen keinen singulären Punkt haben und daher auch nirgends aufhören darf; denn bei Annahme einer solchen singulären Stelle könnten wir in dieselbe den Punkt $O$ verlegen und dies gäbe einen Widerspruch mit unseren früheren Ausführungen, wonach durch $O$ stets zwei reguläre Asymptotencurven hindurchlaufen und eine genügend kleine Umgebung des Punktes $O$ auf unserer Fläche durch regulär verlaufende Asymptotencurven lückenlos erfüllt wird.

Aus diesem Umstande entnehmen wir die analytische Thatsache, dass die Functionen $x, y, z$ für alle reellen $u, v$ eindeutig und unbegrenzt fortsetzbar sind. Um dies sicher zu erkennen, tragen wir vom Punkte $O$ aus auf der Asymptotencurve $v=0$ die Länge $u$ nach der einen oder anderen Richtung hin, je nachdem $u$ positiv oder negativ ist, ab, ziehen durch den erhaltenen Endpunkt die andere Asymptotencurve, tragen dann auf dieser die Länge $v$ nach der einen oder anderen Richtung hin, je nachdem $v$ positiv oder negativ ausfällt, ab und ertheilen endlich dem so erhaltenen Endpunkte, der die rechtwinkligen Coordinaten $x, y, z$ haben möge, die Parameterwerthe $u, v$. Auf diese Weise ist jedem Punkte der $u v$-Ebene jedenfalls ein bestimmter Punkt unserer Fläche zugeordnet und die Funktionen $x, y, z$ die diese Zuordnung vermitteln sind eindeutige für alle reellen Variabeln $u, v$ definirte und reguläre analytische Functionen.

Auch zeigt sich sofort, dass umgekehrt jedem Punkte unserer Fläche mindestens ein Werthepaar $u$, $v$ entspricht. Um dies einzusehen, bezeichnen wir diejenigen Punkte, deren Coordinaten durch Functionswerthe

$$
x(u, v), \quad y(u, v), \quad z(u, v)
$$

dargestellt werden, mit $P$, dagegen die Punkte der Fläche, die durch unsere Abbildung (1) nicht betroffen werden, mit $Q$. Würden nun im Endlichen ein oder mehrere Punkte $Q$ vorhanden sein, so müsste es gewiss mindestens einen Punkt $A$ auf der Fläche geben, in dessen beliebiger Nähe sowohl Punkte $P$ als auch Punkte $Q$ gelegen sind.

Nach den früheren Ausführungen existiren nun für die Umgebung des Punktes $\boldsymbol{A}$ zwei Schaaren von Asymptotencurven, deren jede diese Umgebung einfach und lückenlos überdeckt. Unter diesen Asymptotencurven muss nothwendig mindestens eine solche vorhanden sein, die sowohl einen Punkt $P$ als auch einen Punkt $Q$ enthält. In der That, fassen wir eine der beiden durch $A$ hin durchgehenden Asymptotencurven ins Auge und nehmen wir an dieselbe bestände aus lauter Punkten $P$ (bez. $Q$ ), so würden die Asymptotencurven derjenigen Schaar, zu welcher jene erstere Asymptotencurve nicht gehört, mindestens je einen Punkt $\boldsymbol{P}$ (bez. $Q$ ) nämlich den Schnittpunkt mit der ersteren Asymptotencurve enthal- 
ten. Die sämmtlichen Curven dieser Schaar können aber gewiss nicht aus lauter Punkten $P$ (bez. $Q$ ) bestehen, da sonst die ganze Umgebung von $A$ nur Punkte $P$ (bez. $Q$ ) enthielte.

Es sei nun $l$ die Länge eines Stückes einer Asymptotencurve, deren Anfangspunkt ein Punkt $P$ und deren Endpunkt ein Punkt $Q$ sein möge. Fassen wir die beiden durch den Anfangspunkt $P$ laufenden Asymptotencurven der Fläche ins Auge, so ist jenes Stück von der Länge $l$ nothwendig die Fortsetzung einer dieser beiden Asymptotencurven, und wenn daher $u, v$ die Coordinaten des Anfangspunktes $P$ sind, so wird der Endpunkt jenes Curvenstückes entweder durch die Parameterwerthe $u \pm l, v$ oder $u, v \pm l$ dargestellt-entgegen unserer Annahme derzufolge der Endpunkt $Q$ nicht durch die Formeln (1) darstellbar sein sollte.

Damit ist bewiesen worden, dass durch die Formeln (1) die ganze Fläche zur Darstellung gebracht wird, wenn $u, v$ alle reellen Zahlenwerthe durchläuft.

Endlich ist es für unsere Untersuchung nothwendig einzusehen, dass die Formeln (1) jeden Punkt der Fläche nur durch ein Werthepaar $u$, $v$ darstellen, d. h. dass die gefundene Abbildung (1) unserer Fläche auf die $u v$-Ebene nicht blos für genügend kleine Gebiete sondern im Ganzen genommen eine umkehrbar-eindeutige sein muss.

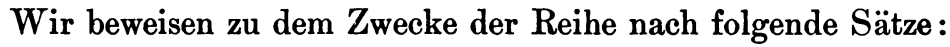

1. Es giebt auf unserer Fläche keine geschlossene, d. h. in sich zurückkehrende Asymptotencurve.

Zum Beweise nehmen wir im Gegentheil an, es sei eine solche Asymptotencurve auf unserer Fläche vorhanden. Wir construire durch jeden Punkt

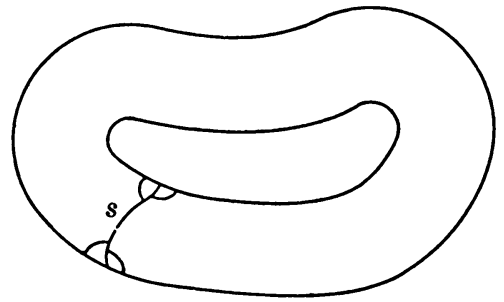

Fig. 2.

derselben die andere Asymptotencurve und tragen auf diesen Curven stets ein Stück $s$ nach derselben Seite hin. Die erhaltenen Endpunkte werden dann entweder eine in sich zurücklaufende Asymptotencurve bilden, oder die Endpunkte des Stückes s beschreiben erst nach zweimaligem Durchlaufen der Grundcurve eine in sich zurückkehrende Asymptotencurve-ein Fall, der eintreten könnte, wenn unsere Fläche eine sogenannte Doppelfläche wäre. Fassen wir nun eine derjenigen Asymptotencurven von der Länge $s$ ins Auge, die uns vorhin zur Construction der neuen geschlossenen Asymptotencurve diente, so bildet dieselbe 
doppelt gerechnet zusammen mit den beiden geschlossenen Asymptotencurven ein Asymptotenviereck, dessen Winkelsumme offenbar genau gleich $2 \pi$ ist. Diese Thatsache aber steht im Widerspruch zu dem vorhin angeführten Satze, wonach der Inhalt eines Asymptotencurvenvierecks stets gleich dem Ueberschuss der Summe seiner Winkel über $2 \pi$ ist und dieser Ueberschuss daher nothwendig positiv sein muss.

2. Irgend zwei durch einen Punkt gehende Asymptotencurven schneiden sich in keinem anderen Punkt unserer Fläche.

Wir denken uns eine Asymptotencurve $a$ nach beiden Richtungen hin ins Unendliche verlängert und dann durch einen Punkt $P_{0}$ derselben nach einer Seite die andere Asymptotencurve $b$ gezogen. Nehmen wir dann im Gegensatz zu unserer Behauptung an, dass diese Asymptotencurve $b$ die ursprüngliche $a$ zum ersten Mal im Punkt $P_{1}$ schnitte, so sind die folgenden zwei Fälle denkbar :

Erstens: die Asymptotencurve $b$ könnte so verlaufen, dass sie in $P_{1}$ von derselben Seite der Asymptotencurve $a$ her eintritt, als sie dieselbe verlassen hat;

Zweitens: die Asymptotencurve $b$ könnte derart verlaufen, dass sie von der anderen Seite der ursprünglichen Asymptotencurve $a$ herkommt und mithin nach Verlassen des Schnittpunktes $\dot{P}_{1}$ nach der nëmlichen Seite der Asymptotencurve $a$ gerichtet ist, wie anfänglich, als sie vom Punkte $P_{0}$ ausging.

Wir wollen zeigen, dass beide Fälle unmöglich sind. Was den ersten Fall betrifft, so bezeichnen wir die Länge der Strecke $P_{0} P_{1}$ auf $a$ mit $l$ und die Mitte dieser Strecke mit $Q$. Sodann denken wir uns durch jeden Punkt der

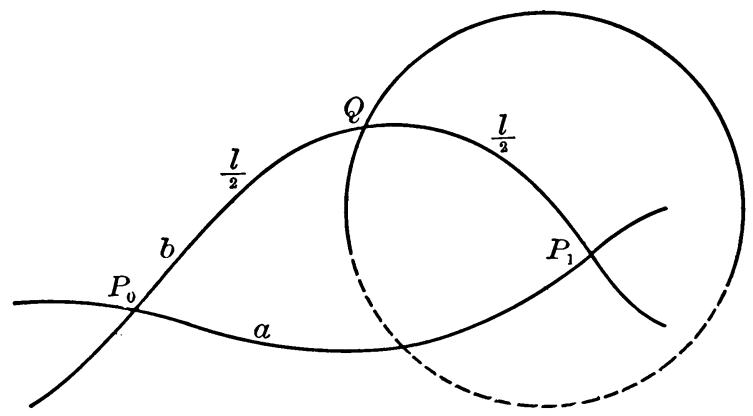

Fra. 3.

Asymptotencurve $a$ die andere Asymptotencurve gezogen und nach der Seite, nach welcher hin die fragliche Strecke $P_{0} P_{1}$ auf $b$ liegt, die Länge $\frac{1}{2} l$ abgetragen. Aus den Punkten $P_{0}$ und $P_{1}$ der Curve $a$ erhalten wir auf diese Weise den nämlichen Punkt $Q$ als Endpunkt. Die sämmtlichen erhaltenen Endpunkte bilden mithin eine Asymptotencurve, welche durch den Punkt $Q$ geht und zu demselben Punkte $Q$ in der nämlichen Richtung zurückkehrt. Dies ist unmöglich, da es nach 1. auf unserer Fläche keine geschlossene Asymptotencurve giebt. 
Damit ist gezeigt, dass der erste Fall nicht stattfinden kann. Aber auch der zweite Fall ist unmöglich. Verliefe nämlich die Asymptotencurve in derWeise, dass sie nach Ueberschreitung des Schnittpunktes $P_{1}$ die nämliche Richtung aufweist, wie früher in $\boldsymbol{P}_{0}$, so könnten wir die Fortsetzung dieses Stückes $P_{0} P_{1}$ der Asymptotencurve $b$ über $P_{1}$ hinaus offenbar dadurch erhalten, dass wir von $P_{0}$ ausgehend durch jeden Punkt des Stückes $P_{0} P_{1}$ von $b$ die andere Asymptotencurve construiren und auf allen diesen Asymptotencurven nach der betreffenden Seite hin das gleiche Stück $P_{0} P_{1}$ der Asymptotencurve $a$ abtragen. Die erhaltenen Endpunkte bilden die Fortsetzung der Asymptotencurve $b$ von $P_{1}$ bis zu einem Punkte $P_{2}$ auf $a$. Aus diesem Stücke $P_{1} P_{2}$ der Asymptotencurve $b$ können wir in gleicher Weise ein neues Stück der Asymptotencurve $b$ construiren, welches über $P_{2}$ hinaus geht und bis zu einem Punkte $P_{3}$ auf $a$ reicht u. s. f. Auch ist klar, wie wir die Asymptotencurve $b$ nach der anderen Richtung hin über $P_{0}$ hinaus durch die entsprechende Construction fortsetzen können und so der Reihe nach zu den Curvenstücken $P_{0} P_{-1}$, $P_{-1} P_{-2}, \cdots$ gelangen. Die Asymptotencurve $b$ schneidet also die Asymptotencurve $a$ in den unendlich vielen gleich weit von einander entfernten Punkten :

$$
\ldots P_{-3}, P_{-2}, P_{-1}, P_{0}, P_{1}, P_{2}, P_{3} \ldots
$$

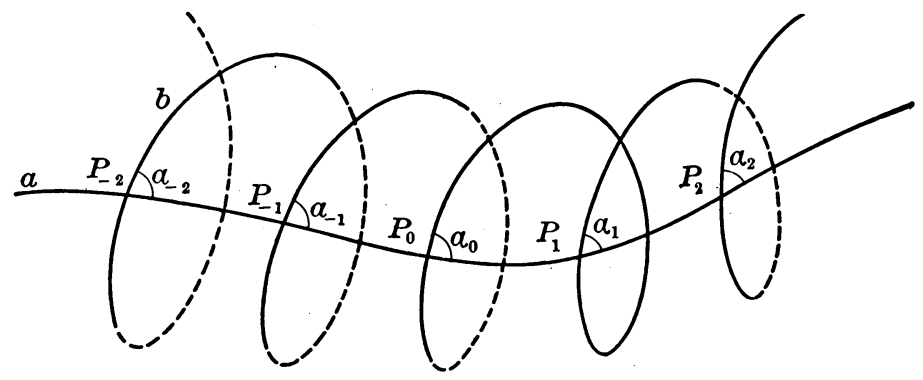

FIG. 4.

Die Winkel, die die Asymptotencurve $b$ mit $a$ in bestimmten Sinne in jenen Schnittpunkten bildet, bezeichnen wir bez. mit

$$
\cdots a_{-3}, a_{-2}, a_{-1}, a_{0}, a_{1}, a_{2}, a_{3} \cdots .
$$

Wir betrachten nun das Asymptotencurvenviereck $P_{0} P_{1} P_{2} P_{1} P_{0}$, welches von den zwei Stücken $P_{0} P_{1}, P_{2} P_{1}$ auf $a$ und den zwei Curvenstücken $P_{1} P_{2}$, $P_{1} P_{0}$ auf $b$ gebildet wird. Die vier Winkel dieses Vierecks sind

$$
a_{0}, \pi-a_{1}, a_{2}, \pi-a_{1},
$$

und da nach dem angeführten Satze über das Asymptotencurvenviereck der Inhalt desselben gleich dem Ueberschuss der Summe seiner Winkel über $2 \pi$ ist und dieser Ueberschuss daher positiv sein muss, so folgt 
d. h.

$$
a_{0}+\pi-a_{1}+a_{2}+\pi-a_{1}>2 \pi,
$$

$$
a_{0}-a_{1}>a_{1}-a_{2}
$$

Ebenso folgt allgemein

$$
a_{k}-a_{k+1}>a_{k+1}-a_{k+2}, \quad(k=0, \pm 1, \pm 2, \cdots) .
$$

Wegen der obigen Ungleichung (4) können jedenfalls $a_{0}-a_{1}$ und $a_{1}-a_{2}$ nicht zugleich 0 sein; wir dürfen die Annahme

$$
a_{0}-a_{1} \neq 0
$$

treffen. Aus (5) folgen die Ungleichungen :

oder

$$
\begin{array}{ll}
a_{-p}-a_{-p+1}>a_{0}-a_{1} & (p=1,2,3, \cdots), \\
a_{0}-a_{1}>a_{p}-a_{p+1} &
\end{array}
$$

Bilden wir die Ungleichungen (6) und (7) für $p=1,2,3, \ldots, n$, so folgt durch Addition derselben leicht

$$
\begin{aligned}
& a_{-n}>a_{0}+n\left(a_{0}-a_{1}\right), \\
& a_{n+1}>a_{1}+n\left(a_{1}-a_{0}\right) .
\end{aligned}
$$

Fällt nun $a_{0}-a_{1}>0$ aus, so ist für genügend grosse Werthe von $n$ jedenfalls die erstere dieser beiden Gleichungen unmöglich, da die Winkel $a_{k}$ sämmtlich kleiner als $\pi$ sind; fällt dagegen $a_{1}-a_{0}>0$ aus, so folgt aus demselben Grunde für genügend grosse Werthe von $n$ die Unmöglichkeit der letzteren Gleichung.

Die Asymptotencurve $b$ darf daher auf keine der beiden angenommenen Arten verlaufen und mithin ist der Beweis für 2. vollständig erbracht.

3. Eine Asymptotencurve unserer Fläche durchsetzt sich selbst an keiner Stelle, d. h. sie besitzt keinen Doppelpunkt.

Zum Beweise nehmen wir im Gegentheil an, es existire eine Asymptotencurve mit einem Doppelpunkt; dann verlegen wir den Anfangspunkt der krummlinigen

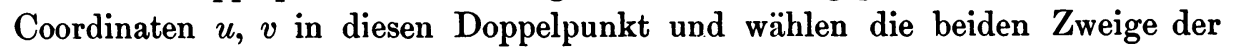
Curvenschleife zu Coordinatenlinien, nach der Schleife hin den positiven Sinn gerechnet.

Wir ziehen jetzt vom Doppelpunkte $(0,0)$ oder vom Punkte $(-s, 0)$ beginnend auf der $u$-Coordinate durch jeden Punkt der Schleife die andere Asymptotencurve und tragen auf dieser nach der positiven Seite hin eine Strecke $s$ ab : wählen wir diese Strecke $s$ genügend klein, so werden die sämmtlichen erhaltenen Endpunkte nach einem oben angeführten Satze über das Asymptotencurvenviereck wiederum eine Asymptotencurve bilden. Diese Asymptotencurve geht vom 
Punkte $(0, s)$ bez. $(-s, s)$ aus, durchsetzt sich selbst im Punkte $(s, s)$ bez. $(-s, s)$ und endigt im Punkte $(s, 0)$ bez. $(-s, 0)$. Wir sehen also, dass die eben construirte Asymptotencurve die ursprüngliche Asymptotencurve in zwei

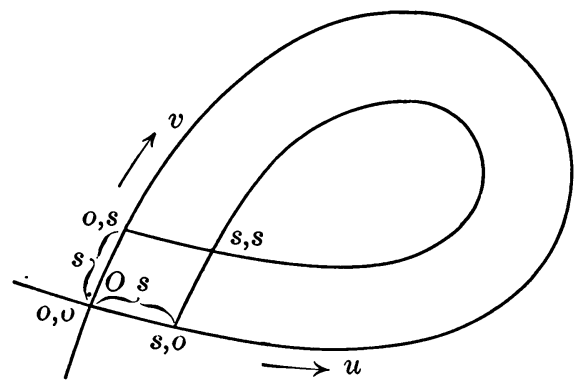

Fig. 5.

verschiedenen Punkten $(0, s)$ und $(s, 0)$ bez. $(0, s)$ und $(-s, 0)$ schneidet; dies ist nach 2. unmöglich.

4. Wenn wir durch jeden Punkt einer Asymptotencurve a die andere Asymptotencurve ziehen und auf dieser nach der nämlichen Seite hin eine bestimmte Strecke s abtragen, so bilden die erhaltenen Endpunkte eine neue Asymptotencurve b, die die ursprüngliche Asymptotencurve a an keiner Stelle schneidet.

Denn wäre $P$ ein Schnittpunkt der Asymptotencurve $b$ mit der ursprünglichen $a$, und tragen wir von $P$ aus auf der Asymptotencurve $b$ die Strecke $s$ nach der betreffenden Seite von $a$ hin ab, so müsste der weitere durch den entstehenden Endpunkt $Q$ hindurchgehende Asymptotencurvenzweig ebenfalls zur Asympto-

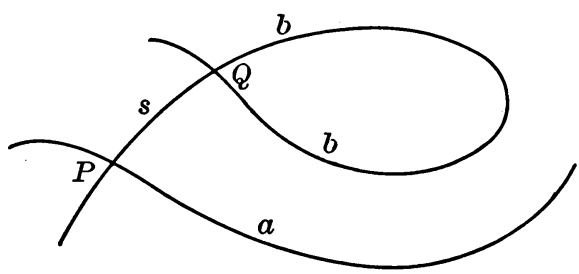

Fig. 6.

tencurve $b$ gehören und mithin wäre $Q$ ein Doppelpunkt der Asymptotencurve $b$; das Auftreten eines Doppelpunktes ist aber nach 3. unmöglich.

Aus den Sätzen 1. bis 4. können wir sofort diese Schlussfolgerungen ziehen :

Die sämmtlichen Asymptotencurven unserer Fläche zerfallen in zwei Schaaren. Irgend zwei derselben Schaar angehörende Asymptotencurven schneiden sich nicht; dagegen schneiden sich je zwei Asymptotencurven, die verschiedenen Schaaren angehören, stets in einem und nur einem Punkte der Fläche.

Die Coordinatenlinien $u=0, v=0$ sind zwei Asymptotencurven die verschiedenen Schaaren angehören. Wegen der Bedeutung der Coordinaten $u, v$ als Längen gewisser Coordinatenabschnitte entnehmen wir aus den eben ausge- 
sprochenen Thatsachen zugleich, dass zu bestimmt gegebenen Werthen von $u, v$ stets nur ein Punkt unserer Fläche gehört d. h. die zu untersuchende Abbildung (1) unserer Fläche auf die uv-Ebene ist nothwendig eine umkehrbar eindeutige. Insbesondere folgt hieraus, dass unsere Fläche einen einfachen Zusammenhang besitzt und keine Doppelfäche ist.

Nachdem wir zu dieser wichtigen Einsicht gelangt sind, berechnen wir den gesammten Inhalt unserer Fläche auf zwei Wegen; wir werden dadurch zu einem Widerspruch gelangen.

Der erstere Weg ist der folgende. Wir betrachten auf unserer Fläche dasjenige aus Asymptotencurven gebildete Viereck, dessen Ecken durch die Coordinaten

$$
u, v ; \quad-u, v ; \quad-u,-v ; \quad u,-v
$$

bestimmt sind. Da jeder Winkel dieses Vierecks $<\pi$ sein muss, so ist die Summe der Winkel des Viereckes jedenfalls $<4 \pi$ und der Inhalt des Viereckes d. h. der Ueberschuss der Summe seiner Windel über $2 \pi$ ist mithin nothwendig $<2 \pi$. Lassen wir nun die Werthe von $u$, $v$ unbegrenzt wachsen, so kommt jeder bestimmte Punkt der Fläche sicher einmal im Inneren eines Viereckes zu liegen und bleibt dann im Inneren aller weiteren Vierecke, so dass das unbegrenzt wachsende Viereck schliesslich die ganze Oberfläche umfasst. Wir entnehmen daraus, dass der Gesammtinhalt unserer Fläche $\leqq 2 \pi$ sein muss.

Andrerseits betrachten wir die geodätischen Linien auf unserer Fläche. Wegen der negativen Krümmung unserer Fläche ist jede geodätische Linie zwischen irgend zweien ihrer Punkte gewiss kürzeste Linie, d. h. von kleinerer Länge als jede andere Linie, die auf der Fläche zwischen den nämlichen zwei Punkten verläuft und sich durch stetige Aenderung in die geodätische Linie überführen lässt. Wir fassen nun irgend zwei vom Punkt $O$ ausgehende geodätische Linien auf unserer Fläche ins Auge und nehmen an, dieselben schnitten sich noch in einem anderen Punkt $P$ der Fläche. Da nach dem oben Bewiesenen unsere Fläche einen einfachen Zusammenhang besitzt, so lässt sich jede dieser beiden geodätischen Linien $O P$ in die andere durch stetige Veränderung überführen; es müsste also nach dem eben Ausgeführten jede derselben kürzer sein als die andere, was nicht möglich ist. Unsere Annahme der Existenz eines Schnittpunktes $P$ ist also zu verwerfen. Durch die nämlichen Schlüsse erkennen wir auch, dass eine geodätische Linie unserer Fläche weder sich durchsetzen noch in sich selbst zurücklaufen darf.

Denken wir uns nun auf allen von $O$ ausgehenden geodätischen Linien die gleiche Länge $r$ abgetragen, so bilden die erhaltenen Endpunkte eine geschlossene doppelpunktslose Curve auf unserer Fläche. Das von dieser Curve umspannte Gebiet besitzt nach den bekannten Formeln der LobatscheFskiJschen Geometrie den Flächeninhalt

$$
\pi\left(e^{\frac{r}{2}}-e^{-\frac{r}{2}}\right)^{2} .
$$


Da dieser Ausdruck für unendlich wachsende Werthe von $r$ selbst über alle Grenzen wächst, so entnehmen wir hieraus, dass auch der Gesammtinhalt unserer Fläche unendlich gross sein müsste. Diese Folgerung steht im Wiederspruch mit der vorhin bewiesenen Thatsache, wonach jener Inhalt stets $\leqq 2 \pi$ ausfallen sollte. Wir sind daher gezwungen, unsere Grundannahme zu verwerfen, d. h. wir erkennen, dass es eine singularitätenfreie und überall regulär analytische Fläche von constanter negativer Krümmung nicht giebt. Inbesondere ist daher auch die zu Anfang aufgeworfene Frage zu verneinen, ob auf die Beltramische Weise die Ganze LobatschefskiJsche Ebene durch eine regulär analytische Fläche im Raume sich verwirklichen lässt.

\section{Ueber Flächen von positiver constanter Krümmung.}

Wir gingen zu Anfang dieser Untersuchung aus von der Frage nach einer Fläche der negativen constanten Krümmung, die überall im Endlichen regulär analytisch verläuft, und gelangten zu dem Resultate, dass es eine solche Fläche nicht giebt. Wir wollen nunmehr mittelst der entsprechenden Methode die gleiche Frage für die positive constante Krümmung behandeln. Offenbar ist die Kugel eine geschlossene singularitätenfreie Fläche der positiven constanten Krümmung, und nach dem von H. LiebmanN * geführten Beweise giebt es auch keine andere geschlossene Fläche von derselben Eigenschaft. Diese Thatsache nun wollen wir aus einem Satze herleiten der von einem beliebigen singularitätenfreien Stücke einer Fläche der positiven constanten Krümmung gilt und folgendermassen lautet.

Auf einer analytischen Fläche der positiven constanten Krümmung +1 sei ein singularitätenfreies einfach oder mehrfach zusammenhängendes Gebiet im Endlichen abgegrenzt: denken wir uns dann in jedem Punkte dieses Gebietes sowie in den Randpunkten desselben die beiden Hauptkrümmungsradien der Fläche construirt, so wird das Maximum der grösseren und folglich auch das Minimum der kleineren der beiden Hauptkrïmmungsradien gewiss in keinem Punkte angenommen, der im Inneren des Gebietes liegt - es sei denn unsere Fläche ein Stïck der Kugel mit dem Radius 1.

Zum Beweise bedenken wir zunächst, dass wegen unserer Voraussetzung das Product der beiden Hauptkrummungsradien überall $=1$ und daher der grössere der beiden Hauptkrümmungsradien stets $\geqq 1$ sein muss. Aus diesem Grunde ist das Maximum der grösseren Hauptkrümmungsradien offenbar nur dann $=1$, wenn beide Hauptkrümmungsradien in jedem Punkte unseres Flächenstückes $=1$ sind. In diesem besonderen Falle ist jeder Punkt des F]ächenstückes ein Nabelpunkt, und man schliesst dann leicht in bekannter Weise, dass das Flächenstück ein Stück der Kugel mit dem Radius 1 sein muss.

*Göttinger Nachrichten, 1899, S. 44.

Trans. Am. Math. Soc. 7 
Nunmehr sei das Maximum der grösseren der beiden Hauptkrümmungsradien unserer Fläche $>1$; dann nehmen wir im Gegensatz zu der Behauptung an, es gäbe im Inneren des Flächenstückes einen Punkt $O$, in welchem jenes Maximum stattfinde. Da dieser Punkt $O$ gewiss kein Nabelpunkt sein kann und überdies ein regulärer Punkt unserer Fläche ist, so wird die Umgebung dieses Punktes lückenlos und einfach von jeder der beiden Schaaren von Krümmungslinien der beiden Flächen bedeckt. Benutzen wir diese Krümmungslinien als Coordinatenlinien und den Punkt $O$ selbst als Anfangspunkt des krummlinigen Coordinatensystems, so gelten nach der bekannten Theorie der Flächen positiver constanter Krümmung die folgenden Thatsachen.*

Es bedeute $r_{1}$ den grösseren der beiden Hauptkrümmungsradien für den Punkt $(u, v)$ in der Umgebung des Anfangspunktes $O=(0,0)$; es ist in dieser Umgebung $r_{1}>1$. Man setze

$$
\rho=\frac{1}{2} \log \frac{r_{1}+1}{r_{1}-1}
$$

dann genügt die positive reelle Grösse $\rho$ als Function von $u$, $v$ der partiellen Differentialgleichung

$$
\frac{\partial^{2} \rho}{\partial u^{2}}+\frac{\partial^{2} \rho}{\partial v^{2}}=\frac{e^{-2 \rho}-e^{2 \rho}}{4}
$$

Da bei abnehmendem $r_{1}$ die Function $\rho$ nothwendig wächst, so muss $\rho$ als Function von $u, v$ an der Stelle $u=0, v=0$ einen Minimalwerth aufweisen, und demnach hat die Entwickelung von $\rho$ nach Potenzen der Variabeln $u, v$ nothwendig die Gestalt

$$
\rho=a+a u^{2}+2 \beta u v+\gamma v^{2}+\cdots,
$$

wo $a, a, \beta, \gamma$ Constante bedeuten und dabei die quadratische Form

$$
a u^{2}+2 \beta u v+\gamma v^{2},
$$

für reelle $u, v$ niemals negative Werthe annehmen darf. Aus letzterem Umstande folgen für die Constanten $a$ und $\gamma$ nothwendig die Ungleichungen :

$$
a \geqq 0 \text { und } \gamma \geqq 0 \text {. }
$$

Andrerseits wollen wir die Entwicklung für $\rho$ in die Differentialgleichung (8) einsetzen : für $u=0, v=0$ erhalten wir dann

$$
2(a+\gamma)=\frac{e^{-2 a}-e^{2 a}}{4}
$$

Da die Constante $a$ den Werth von $\rho$ im Punkte $O=(0,0)$ darstellt und mithin

*DARBoux, Leģons sur la theorie générale des surfaces, Bd. 3, No. 776.

BIANCHI, Lezioni di geometria differenziale, § 264. 
positiv ausfällt, so ist hier der Ausdruck rechter Hand jedenfalls $<0$; die letztere Gleichung führt desshalb zu der Ungleichung

$$
a+\gamma<0,
$$

welche mit den Ungleichungen (9) in Widerspruch steht. Damit ist unsere ursprüngliche Annahme, wonach die Stelle des Maximums im Inneren des Flächenstückes liege als unzutreffend und mithin der oben aufgestellte Satz als richtig erkannt.

Der eben bewiesene Satz lehrt offenbar folgende Thatsache. Wenn wir aus der Kugeloberfläche ein beliebiges Stück ausgeschnitten denken und dann dieses Stück beliebig verbiegen, so findet sich das Maximum aller grösseren vorkommenden Hauptkrümmungsradien stets auf dem Rande des Flächenstückes. Eine geschlossene Fläche besitzt keinen Rand und daraus folgt, wie bereits oben bemerkt, sofort der Satz, das eine geschlossene analytische singularitätenfreie Fläche mit der positiven constanten Krümmung 1 stets die Kugel mit dem Radius 1 sein muss. Dieses Resultat drückt zugleich aus, dass man die Kugel als Ganzes nicht verbiegen kann, ohne den regulär analytischen Charakter der Fläche irgendwo zu stören.

GötTinaen, 1900. 